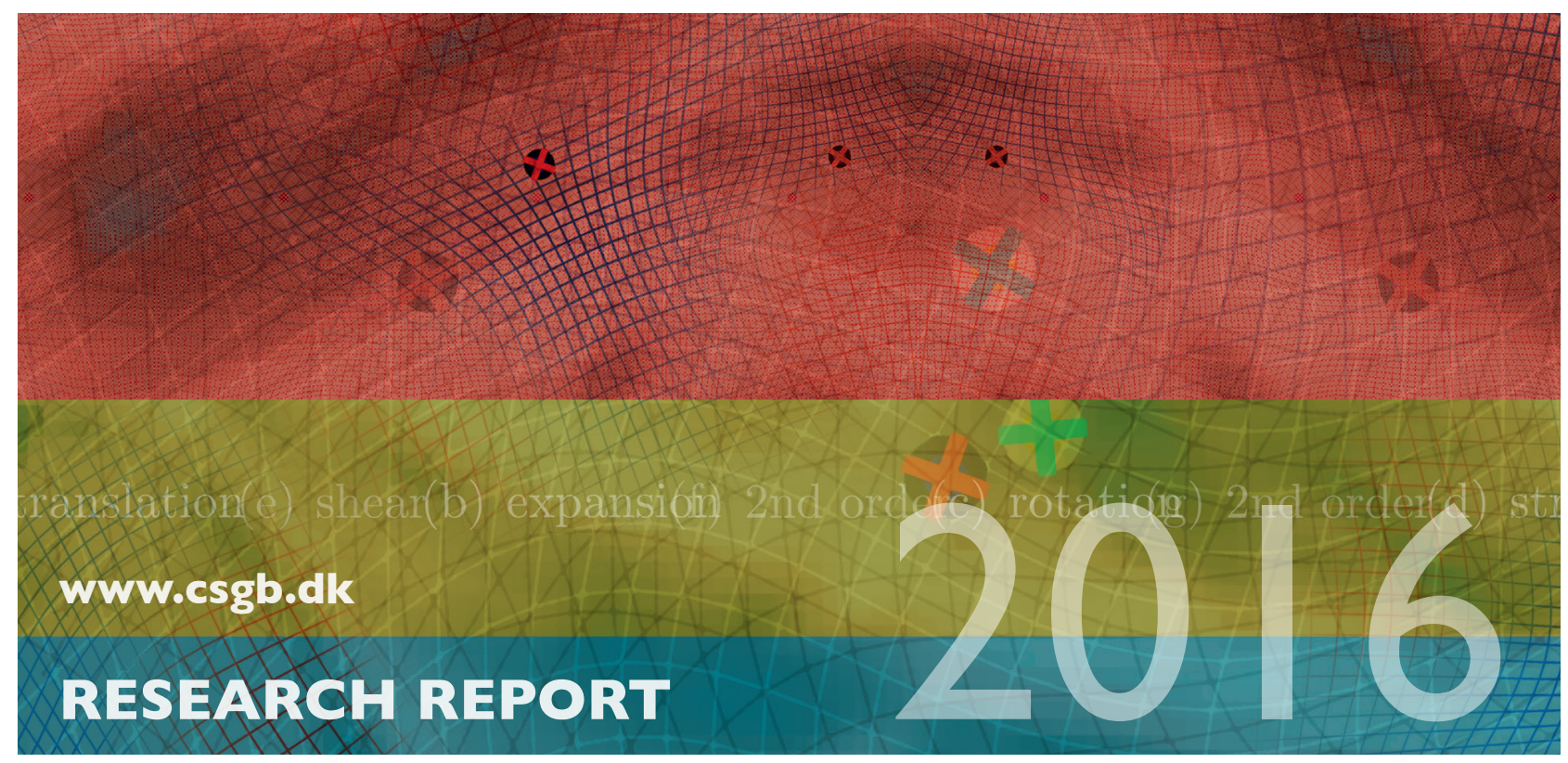

Anne Marie Svane

Valuations in Image Analysis

No. 04, February 2016 


\title{
Valuations in Image Analysis
}

\author{
Anne Marie Svane \\ Department of Mathematics \\ Aarhus University \\ amsvane@math.au.dk
}

\begin{abstract}
When intrinsic volumes and Minkowski tensors of a real world structure are computed, this is often based on a digital image. The digitization causes some estimation problems due to the anisotropic nature of the digital grid. Even the most natural and frequently used algorithms based on counting the local pixel/voxel configurations are often biased. In this chapter, we survey the known results on convergence of these local algorithms with a focus on estimation of intrinsic volumes. Moreover, we present some of the latest attempts to define convergent algorithms.
\end{abstract}

\section{Introduction}

Consider an object $X \subseteq \mathbb{R}^{n}$. To describe its geometry, we can look at its intrinsic volumes $V_{0}(X), \ldots, V_{n}(X)$. These include such important characteristics as volume $V_{n}$, surface area $2 V_{n-1}$, integrated mean curvature $2 \pi(n-1)^{-1} V_{n-2}$, and Euler characteristic $V_{0}$. (See [17] for more details.) The intrinsic volumes are all rotation and and translation invariant. Non-invariant properties, such as position, orientation, and elongation, are captured by the Minkowski tensors. The $r$ th Minkowski volume tensor for $r \geq 0$ is an element of $\left(\mathbb{R}^{n}\right)^{\otimes r}$ given by

$$
\Phi_{n}^{r, 0}(X)=\frac{1}{r !} \int_{X} x^{r} d x
$$

where $x^{r}$ is the $r$-fold tensor product of $x$. Moreover, for $r, s \geq 0$ and $0 \leq m \leq n-1$, there is a Minkowski tensor $\Phi_{m}^{r, s}(X) \in\left(\mathbb{R}^{n}\right)^{\otimes(r+s)}$ defined by

$$
\Phi_{m}^{r, s}(X)=\frac{1}{r ! s !} \frac{\omega_{n-m}}{\omega_{n-m+s}} \int_{\mathbb{R}^{n} \times S^{n-1}} x^{r} u^{s} \Lambda_{m}(X ; d(x, u)) .
$$

Here $S^{n-1}$ is the unit sphere in $\mathbb{R}^{n}$ and $\omega_{n}$ is its surface area. Moreover, $\Lambda_{m}(X ; \cdot)$ is the $m$ 'th generalized curvature measure on $\mathbb{R}^{n} \times S^{n-1}$, see [17] when $X$ is convex and [27] for more general set classes. The integrand $x^{r} u^{s}$ is the symmetric tensor product of $r$ copies of $x$ and $s$ copies of $u$. Intrinsic volumes are special cases of 
the Minkowski tensors since $\Phi_{m}^{0,0}(X)=V_{m}(X)$. (For more on Minkowski tensors, see $[17]$.)

As described in [18] and [19], Minkowski tensors are useful tools for physicists to characterize geometric properties of a material. The tensors are often computed based on a digital image, for instance from a microscope or a scanner. This causes several problems. Not only are such images often blurred and noisy, the digitization itself may also introduce a bias. This is the topic of the present chapter.

A digital image is divided into pixels or voxels and the object is measured inside each. The pixel (voxel) midpoints form a lattice. Gaining information about the underlying object can thus be considered a stereological type of problem, but where the object is only known along a lattice rather than on an affine plane. In stereology, unbiased estimators for intrinsic volumes and, more generally, Minkowski tensors can be obtained by randomizing the rotation and translation of the intersection plane. In image analysis, it is often not possible to rotate the observation lattice. This causes a rotation bias in many algorithms.

Another problem is that, while the boundary of the object is still visible on lower dimensional planes, a lattice will most likely not hit any boundary points and the boundary can behave wildly between the lattice points. This makes it hard to estimate boundary properties. In grey-scale images, the object boundary is represented by a blurred zone around the true boundary. As we shall see, this makes boundary estimation easier.

A third problem is that the data amount is often large. Therefore, fast algorithms are required. The focus of this chapter will therefore be on the development of algorithms with low computation time.

We are going to review some of the mathematical results on digital estimators for intrinsic volumes and Minkowski tensors. In Section 2, we consider the ideal situation where there is no noise or blurring. The emphasis will be on the so-called local algorithms, which are the most frequently occurring ones, but some global methods will also be discussed. In Section 3, we consider the situation where the digital image is blurred.

\section{Digital algorithms for black-and-white images}

In this section we consider the ideal situation where the digital image is sharp and noise-free. A mathematical model for such an image is given in Section 2.1. Local algorithms are defined and discussed in Section 2.2. Other classes of algorithms are described in Section 2.3.

\subsection{Black-and-white images}

Let $X \subseteq \mathbb{R}^{n}$ be the object that we are trying to observe. A digital image is divided into pixels (voxels). If there is no noise or blurring, we can measure exactly whether or not each pixel midpoint belongs to $X$. A pixel (voxel) is colored black if its midpoint lies in $X$ and white otherwise. This is illustrated in Figure 1.

If we let $\mathbb{L}$ denote the lattice formed by the pixel (voxel) midpoints, then the information contained in a black-and-white image corresponds to the set $X \cap \mathbb{L}$ of 

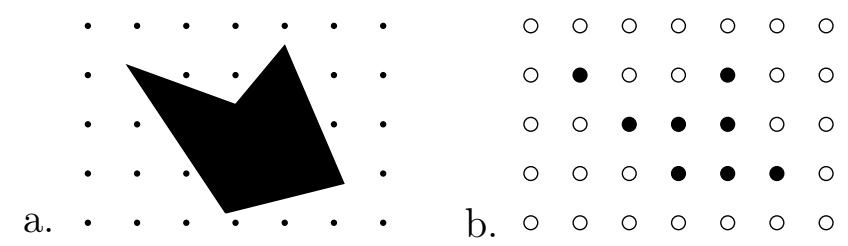

Figure 1: Example of a black-and-white image. Figure a. shows the object together with the grid of pixel midpoints. Figure b. shows the resulting digital image.

black pixel midpoints. We will assume throughout that $X$ is compact and topologically regular, i.e., $X$ is the closure of its own interior. This ensures that $X$ does not have any lower dimensional parts that we are not able to see in the image.

Clearly, there is not enough information in $X \cap \mathbb{L}$ to determine the Minkowski tensors. But going to a higher resolution will give us more information about $X$. This corresponds to scaling $\mathbb{L}$ by a small factor $a>0$, resulting in the image $X \cap a \mathbb{L}$.

In most applications, $\mathbb{L}$ is the standard lattice $\mathbb{Z}^{n}$ or a rotation and translation of this. However, other cases can occur, for instance the hexagonal lattice in 2D [14]. For this reason, we let $\mathbb{L}$ be arbitrary.

\subsection{Local algorithms for intrinsic volumes}

The most popular type of algorithms for estimating intrinsic volumes and Minkowski tensors is the class of so-called local algorithms. The reason for the name is that the algorithm only depends on what the image looks like locally.

The intuition behind is the additivity of Minkowski tensors: By the inclusionexclusion formula [17, Eq. (3.4.3)], they can be computed as a sum of contributions from each $k \times \cdots \times k$ lattice cell depending only on the intersection of $X$ with that cell. Since the only thing we know about $X$ in each cell is the configuration of black and white points, we estimate the contribution from each cell by a so-called weight depending only on the configuration. The Minkowski tensor is then estimated by counting the number of occurrences of each possible $k \times \cdots \times k$ configuration of black and white points in the image and taking a weighted sum of configuration counts.

Since such an algorithm only requires reading through the image once, they are very fast. This makes local algorithms a popular choice in applications. Moreover, geometric intuition can give an idea about how to choose the weights.

\subsubsection{Definition of local algorithms}

To give a precise definition of local algorithms, we first introduce some notation: Suppose the lattice is given by $\mathbb{L}=A\left(\mathbb{Z}^{n}+c\right)$ where $A \in \operatorname{Gl}(n)$ and $c \in[0,1)^{n}$. The fundamental lattice $k$-cell of $\mathbb{L}$ is $C_{0}^{k}:=A\left([0, k)^{n}\right)$. The volume of $C_{0}^{1}$ is denoted $c_{\mathbb{L}}$. The set of lattice points in $C_{0}^{k}$ is denoted by $C_{0,0}^{k}:=C_{0}^{k} \cap \mathbb{L}$. The translation of $C_{0,0}^{k}$ by $z \in \mathbb{R}^{n}$ is denoted by $C_{z, 0}^{k}:=C_{0,0}^{k}+z$.

A $k \times \cdots \times k$ configuration is a partition of $C_{0,0}^{k}$ into two disjoint sets $B$ (black points) and $W$ (white points). We denote the $2^{k^{n}}$ possible $k \times \cdots \times k$ configurations 


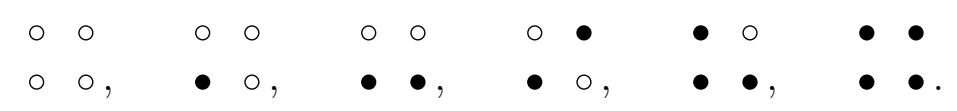

Figure 2: The possible $2 \times 2$ configurations in $2 \mathrm{D}$.

by $\left(B_{l}, W_{l}\right)$ for $l=1, \ldots, 2^{k^{n}}$. For instance, up to rotation there are six possible $2 \times 2$ configurations in 2D. These are shown in Figure 2.

Let $N_{l}(X \cap a \mathbb{L})$ be the number of occurrences of the configuration $\left(B_{l}, W_{l}\right)$ in the image, i.e.

$$
N_{l}(X \cap a \mathbb{L})=\sum_{z \in a \mathbb{L}} \mathbf{1}_{\left\{z+a B_{l} \subseteq X,\left(z+a W_{l}\right) \cap X=\emptyset\right\}} .
$$

We estimate $V_{m}(X)$ by a weighted sum of these configuration counts:

Definition 1. A local algorithm for the intrinsic volume $V_{m}$ is an algorithm of the form

$$
\hat{V}_{m}(X)=a^{m} \sum_{l=1}^{2^{k^{n}}} w_{l}^{(m)} N_{l}(X \cap a \mathbb{L}),
$$

where $w_{l}^{(m)}$ can be arbitrary real numbers, referred to as the weights.

Many natural approaches to defining digital estimators result in a local algorithm. The most simple one is based on approximating $X$ by

$$
\hat{X}=\bigcup_{z \in X \cap a \mathbb{L}}\left(z+a C_{0}^{1}\right)
$$

see [13, Section 2.3.1]. Then $V_{m}(\hat{X})$ can be used as an estimate for $V_{m}(X)$. The intrinsic volumes of $\hat{X}$ can be computed by a local algorithm. This can easily be realized by applying the inclusion-exclusion formula to $\hat{X}$. Local algorithms based on different reconstructions of $X$ have also been considered, see e.g. [13, 15].

Other approaches are inspired by integral geometry. These take as a starting point the Steiner formula [12] or a discretization of the Crofton formula [14, 15]. The results are again local algorithms.

\subsubsection{Convergence in the design-based setting}

To evaluate the quality of an algorithm, it is often tested in a design based setting. This means that the object $X$ is considered as deterministic, whereas the lattice is randomized. In this section, we consider what we will call a stationary lattice, that is, a lattice of the form $\mathbb{L}=\mathbb{L}_{0}+c$, where $\mathbb{L}_{0}$ is a fixed lattice and $c \in C_{0}^{1}$ is a uniform random translation vector. In applications, this is often the only randomization that is possible. A reasonable criterion for an algorithm is unbiased in this setting, at least asymptotically when the resolution tends to infinity we would like that $\lim _{a \rightarrow 0} \mathbf{E} \hat{V}_{n}(X)=V_{m}(X)$.

There is a simple estimator for the volume of $X$ that is unbiased even in finite resolution: 
Theorem 2. The well-known volume estimator that counts the number of black lattice points is a local algorithm with $k=1$ given by

$$
\hat{V}_{n}(X)=a^{n} c_{\mathbb{L}} \sum_{z \in a \mathbb{L}} \mathbf{1}_{\{z \in X \cap a \mathbb{L}\}} .
$$

This algorithm satisfies

$$
\mathbf{E} \hat{V}_{n}(X)=V_{n}(X) .
$$

Note that the algorithm in Theorem 2 is actually computing the volume of the approximating set $\hat{X}$ defined in (2.2).

In $2 \mathrm{D}$ and $3 \mathrm{D}$, the Euler characteristic $V_{0}(X)$ can also be estimated by $V_{0}(\hat{X})$. This requires the following smoothness condition on the boundary:

Definition 3. A set $X \subseteq \mathbb{R}^{n}$ is called $r$-regular if for every boundary point $x \in \operatorname{bd} X$, there are two balls of radius $r$ containing $x$ whose interiors are completely contained in $X$ and $\mathbb{R}^{n} \backslash X$, respectively.

The following theorem is proved in 2D in [16] and in 3D in [3]:

Theorem 4. Let $X$ be an $r$-regular subset of $\mathbb{R}^{2}$ or $\mathbb{R}^{3}$. Then for a sufficiently small compared to $r$,

$$
V_{0}(\hat{X})=V_{0}(X)
$$

Unfortunately, the estimator $V_{m}(\hat{X})$ is not unbiased for $1 \leq m \leq n-1$, not even when $a \rightarrow 0$. This is part of a more general phenomenon. Even when the underlying set is a convex polytope, the following was proved in [23], see also [8] when $k=2$ :

Theorem 5. There exists no estimator of the form (2.1) for $V_{m}$ with $m<n$ that is asymptotically unbiased for all compact convex polytopes with non-empty interior.

In fact, there is a measure $\nu$ on the set $\mathcal{P}_{0}^{n}$ of compact convex polytopes with non-empty interior, such that any local algorithm for $V_{m}, m<n$, is biased on a set of polytopes with positive $\nu$-measure. See [23] for details.

Moreover, for $0 \leq m \leq n-2$, it can be shown that the worst case bias

$$
\sup _{X \in \mathcal{P}_{0}^{n}}\left|\frac{\lim _{a \rightarrow 0} \hat{V}_{m}(X)-V_{m}(X)}{V_{m}(X)}\right|
$$

is always at least $100 \%$. For surface area in 3D, i.e. when $m=2$ and $n=3$, one can do a bit better. It was shown in [28] that the worst case bias is always at least $4 \%$. The authors give an explicit algorithm that minimizes the bias.

Theorem 4 showed that under certain smoothness assumptions on the boundary, estimation of the Euler characteristic is possible. This is not the case in general. The following was proved in [23]:

Theorem 6. Let $m>0$ and $m=n-1$ or $m=n-2$. There exists no estimator for $V_{m}$ of the form (2.1) that is asymptotically unbiased for all $r$-regular sets. 
To prove these theorems, it is necessary to study the mean $\mathbf{E} \hat{V}_{m}(X)$, which is a linear combination of the mean configuration counts $\mathbf{E} N_{l}(X \cap a \mathbb{L})$. A simple computation shows that

$$
\begin{aligned}
\mathbf{E} N_{l}(X \cap a \mathbb{L}) & =\mathbf{E}\left(\sum_{z \in a \mathbb{L}} \mathbf{1}_{\left\{z+a B_{l} \subseteq X,\left(z+a W_{l}\right) \cap X=\emptyset\right\}}\right) \\
& =\sum_{z \in a \mathbb{L}_{0}} \int_{C_{0}^{1}} \mathbf{1}_{\left\{z+a c+a B_{l} \subseteq X,\left(z+a c+a W_{l}\right) \cap X=\emptyset\right\}} d c \\
& =a^{-n} \int_{\mathbb{R}^{n}} 1_{\left\{z+a B_{l} \subseteq X,\left(z+a W_{l}\right) \cap X=\emptyset\right\}} d z .
\end{aligned}
$$

If $X$ is $r$-regular and $f$ is a function supported within distance $r$ from bd $X$, then the generalized Steiner formula of [7] yields the following formula for the integral

$$
\int_{\mathbb{R}^{n}} f(x) d x=\sum_{i=0}^{n-1} \int_{\mathrm{bd} X} \int_{-r}^{r} t^{i} f(x+t u(x)) d t \mu_{i}(d x),
$$

where the $\mu_{i}$ are signed measures on bd $X$ and $u(x)$ is the outward pointing normal vector at $x \in \operatorname{bd} X$.

If $B_{l}$ and $W_{l}$ are both non-empty, then all points $z$ satisfying $z+a B_{l} \subseteq X$ and $\left(z+a W_{l}\right) \cap X=\emptyset$ lie at distance at most $a k \sqrt{n}$ from the boundary, so the generalized Steiner formula can be applied to $\mathbf{1}_{\left\{z+a B_{l} \subseteq X,\left(z+a W_{l}\right) \cap X=\emptyset\right\}}$ when $a$ is small. Thus, to determine the asymptotic behavior of (2.3), one must study the function $\mathbf{1}_{\left\{z+a B_{l} \subseteq X,\left(z+a W_{l}\right) \cap X=\emptyset\right\}}$ along the normal lines $x+t u(x)$. This idea first appeared in [10] and was extended in [22].

\subsubsection{Local algorithms for Minkowski tensors}

Local algorithms for Minkowski 2-tensors have been suggested in [18, 19]. Since these are position dependent, the weights in Definition 1 will generally have to depend on position, i.e. we must consider algorithms of the form

$$
\sum_{l} \sum_{z \in a \mathbb{L}} w_{l}(z, a) \mathbf{1}_{\left\{z+a B_{l} \subseteq X,\left(z+a W_{l}\right) \cap X=\emptyset\right\}},
$$

where $w_{l}: \mathbb{R}^{n} \times(0, \infty) \rightarrow\left(\mathbb{R}^{n}\right)^{\otimes(r+s)}$ depends on position and resolution.

Estimation of volume tensors is easy, since a Riemann sum

$$
\hat{\Phi}_{n}^{r, 0}(X)=a^{n} c_{\mathbb{L}} \frac{1}{r !} \sum_{z \in X \cap a \mathbb{L}} z^{r}
$$

yields an unbiased local algorithm.

There are no convergence results in the literature about local algorithms for other Minkowski tensors than intrinsic volumes, but asymptotic formulas for the mean of a local algorithm taking position into account could easily be derived (see [5] in the case of Boolean models). Apart from the most trivial tensors, asymptotically unbiased local estimators for Minkowski tensors are not expected to exist. 


\subsubsection{Isotropic lattices}

One could also consider a version of the design based setting where the lattice is both randomly translated and rotated. That is, we consider the lattice $R(\mathbb{L}+c)$ where the translation vector $c \in C_{0}^{1}$ and the rotation $R \in \mathrm{SO}(n)$ are both uniform random and mutually independent. We say that $\mathbb{L}$ is stationary isotropic. In this setting, asymptotically unbiased estimators do exist [22]:

Theorem 7. If $X$ is r-regular and $\mathbb{L}$ is stationary isotropic, then there exist local algorithms for $V_{m}$ with $m=n, n-1, n-2$ that are asymptotically unbiased, i.e.

$$
\lim _{a \rightarrow 0} \mathbf{E} \hat{V}_{m}(X)=V_{m}(X) .
$$

Explicit algorithms are given in [22].

If the algorithms are applied to a stationary isotropic Boolean model with a fixed lattice, a similar result seems to hold: There exists asymptotically unbiased estimators for $V_{n}, V_{n-1}$, and $V_{n-2}$. At least, this has been shown in both 2D [21] and 3D [5]. Again, isotropy is essential.

These results suggest that it is the lack of isotropy of $\mathbb{L}$ that causes the bias in the results of Section 2.2.2.

\subsubsection{Variance of the local volume estimator}

We consider again the volume estimator given by lattice point counting

$$
\hat{V}_{n}(X)=a^{n} c_{\mathbb{L}} \sum_{z \in a \mathbb{L}} \mathbf{1}_{X}(z)
$$

from Theorem 2. While this has the correct mean in the design based setting, determining the exact error $\left|\hat{V}_{n}(X)-V_{n}(X)\right|$ is a classical and difficult topic. Even when $X$ is the unit ball centered at the origin, the optimal bound on the error when $a \rightarrow 0$ is unknown. This is known as the Gauss circle problem.

Instead, we will consider the variance in the design based setting where $\mathbb{L}$ is stationary and isotropic. To study the variance, we first consider $\mathbf{E}\left(\hat{V}_{n}(X)^{2}\right)$ :

$$
\begin{aligned}
\mathbf{E}\left(\hat{V}_{n}(X)^{2}\right) & =a^{2 n} c_{\mathbb{L}}^{2} \mathbf{E}\left(\sum_{z \in a \mathbb{L}} \mathbf{1}_{X}(z)\right)^{2} \\
& =a^{2 n} c_{\mathbb{L}}^{2} \int_{\mathrm{SO}(n)} \int_{C_{0}^{1}}\left(\sum_{z_{1} \in a R \mathbb{L}_{0}} \sum_{z_{2} \in a R\left(\mathbb{L}_{0}+c\right)} \mathbf{1}_{X}\left(z_{2}\right) \mathbf{1}_{X}\left(z_{2}+z_{1}\right)\right) d c d R \\
& =a^{n} c_{\mathbb{L}} \int_{\mathrm{SO}(n)}\left(\sum_{z_{1} \in \mathbb{L}_{0}} \int_{\mathbb{R}^{d}} \mathbf{1}_{X}\left(R z_{2}\right) \mathbf{1}_{X}\left(R\left(z_{2}+a z_{1}\right)\right) d z_{2}\right) d R \\
& =a^{n} c_{\mathbb{L}} \int_{\mathrm{SO}(n)}\left(\sum_{z_{1} \in \mathbb{L}_{0}} \mathbf{1}_{X} *\left(\mathbf{1}_{X}\right)^{-}\left(-a R z_{1}\right) d R\right),
\end{aligned}
$$

where $g^{-}$denotes the function $x \mapsto g(-x)$. The Poisson summation formula [20, 
VII, Cor. 1.8] yields:

$$
\begin{gathered}
a^{n} c_{\mathbb{L}} \int_{\mathrm{SO}(n)}\left(\sum_{z \in \mathbb{L}_{0}} \mathbf{1}_{X} *\left(\mathbf{1}_{X}\right)^{-}(-a R z)\right) d R=\sum_{\xi \in \mathbb{L}_{0}^{*}} \int_{\mathrm{SO}(n)} \mathcal{F}\left(\mathbf{1}_{X} *\left(\mathbf{1}_{X}\right)^{-}\right)\left(a^{-1} R \xi\right) d R \\
=\sum_{\xi \in \mathbb{L}_{0}^{*}} \int_{\mathrm{SO}(n)}\left|\mathcal{F}\left(\mathbf{1}_{X}\right)\left(a^{-1} R \xi\right)\right|^{2} d R=\omega_{n}^{-1} \sum_{\xi \in \mathbb{L}_{0}^{*}} \int_{S^{n-1}}\left|\mathcal{F}\left(\mathbf{1}_{X}\right)\left(a^{-1}|\xi| u\right)\right|^{2} d u .
\end{gathered}
$$

Here $\mathcal{F}$ denotes the Fourier transform and $\mathbb{L}_{0}^{*}$ is the so-called dual lattice of $\mathbb{L}_{0}$. We have used the fact that the Fourier transform of a convolution is a product of Fourier transforms and that $\mathcal{F}\left(g^{-}\right)$is the complex conjugate of $\mathcal{F}(g)$. Recalling that

$$
\mathcal{F}\left(\mathbf{1}_{X}\right)(0)=\int_{\mathbb{R}^{n}} \mathbf{1}_{X}(x) d x=\mathbf{E}\left(\hat{V}_{n}(X)\right),
$$

we find

$$
\begin{aligned}
\operatorname{var}\left(\hat{V}_{n}(X)\right) & =\mathbf{E}\left(\hat{V}_{n}(X)^{2}\right)-\mathbf{E}\left(\hat{V}_{n}(X)\right)^{2} \\
& =\omega_{n}^{-1} \sum_{\xi \in \mathbb{L}_{0}^{*} \backslash\{0\}} \int_{S^{n-1}}\left|\mathcal{F}\left(\mathbf{1}_{X}\right)\left(a^{-1}|\xi| u\right)\right|^{2} d u
\end{aligned}
$$

It was shown in [1] that if $X$ is a smooth manifold, then for $a^{-1}|\xi|$ sufficiently large,

$$
\int_{S^{n-1}}\left|\mathcal{F}\left(\mathbf{1}_{X}\right)\left(a^{-1}|\xi| u\right)\right|^{2} d u \leq C(X) a^{d+1}|\xi|^{-d-1},
$$

where $C(X)>0$ is a constant depending on $X$. It follows that:

Theorem 8. If $X$ is a smooth manifold, then for a sufficiently small

$$
\operatorname{var}\left(\hat{V}_{n}(X)\right) \leq a^{d+1} \omega_{n}^{-1} C(X) \sum_{\xi \in \mathbb{L}_{0}^{*} \backslash\{0\}}|\xi|^{-d-1} .
$$

Getting a precise formula for the variance is not possible. When $X$ is smooth and convex with nowhere vanishing Gauss curvature, there are formulas for the Fourier coefficients. These show that each term $\int_{S^{n-1}}\left|\mathcal{F}\left(\mathbf{1}_{X}\right)\left(a^{-1}|\xi| u\right)\right|^{2} d u$ oscillates between 0 and $8 V_{n-1}(X) a^{d+1}|\xi|^{-d-1}$, see [4]. It is therefore hard to determine the sum (2.4).

If the underlying set is a random set $\mathbf{X}$, it is sometimes possible to obtain precise formulas for the asymptotic variance. Under suitable conditions on $\mathbf{X}$, it is shown in [11] that

$$
\lim _{a \rightarrow 0} a^{-d-1} \operatorname{var}\left(\hat{V}_{n}(\mathbf{X})\right)=4 \omega_{n}^{-1} \mathbf{E} V_{n-1}(\mathbf{X}) \sum_{\xi \in \mathbb{L}_{0}^{*}}|\xi|^{-d-1}
$$

\subsection{Other types of algorithms}

Despite the negative convergence results, local algorithms are still being used because of their low computation time. But there are also various attempts in the literature to define algorithms that take the global structure of the image into account without loosing too much speed. 
In [9], a semi-local algorithm is suggested for estimation of Euler characteristic. Assume that $X$ is known to belong to the class $\mathcal{K}_{0}^{n}$ of compact convex sets with nonempty interior. Generally, $\hat{X}$ does not have the same in Euler characteristic as $X$, but it is shown in [9] that after throwing away certain connected components of $\hat{X}$ in a systematic way, it does. The computation time of this algorithm is also linear in the number of pixels (voxels).

There are also convergent algorithms for the remaining $\Phi_{m}^{r, s}$ when $X \in \mathcal{K}_{0}^{n}$. The convex hull of $X \cap a \mathbb{L}, \operatorname{conv}(X \cap a \mathbb{L})$, converges to $X$ in the Hausdorff metric when $a \rightarrow 0$, see [6]. The Minkowski tensors are continuous with respect to the Hausdorff metric, so

$$
\lim _{a \rightarrow 0} \Phi_{m}^{r, s}(\operatorname{conv}(X \cap a \mathbb{L}))=\Phi_{m}^{r, s}(X) .
$$

Hence $\Phi_{m}^{r, s}(\operatorname{conv}(X \cap a \mathbb{L}))$ can be taken as an estimate for $\Phi_{m}^{r, s}(X)$. The optimal computation time for the convex hull of a set of $N$ points is $\mathrm{O}\left(N \log N+N^{\lfloor n / 2\rfloor}\right)$, see $[2]$.

The method of convex hulls does obviously not generalize to non-convex sets. Another approach [6] is based on computing the Voronoi cells of $X \cap a \mathbb{L}$. The optimal computation time for the Voronoi cells of $N$ points is almost as good as for the convex hull, namely $\mathrm{O}\left(N \log N+N^{\lceil n / 2\rceil}\right)$, see [2]. This algorithm applies to all sets of positive reach:

Definition 9. Let $X \subseteq \mathbb{R}^{n}$ and $R \geq 0$. Then $X^{R}=\left\{x \in \mathbb{R}^{n} \mid d(x, X) \leq R\right\}$ denotes the parallel set of $X$. The reach of $X, \operatorname{Reach}(X)$, is the supremum over all $R \geq 0$ for which every point in $X^{R}$ has a unique nearest point in $X$. If $\operatorname{Reach}(X)>0$, then we say that $X$ has positive reach.

The idea is to define the Voronoi tensor of a set $Y \subseteq \mathbb{R}^{n}$ for each pair $r, s \geq 0$ by

$$
\mathcal{V}_{R}^{r, s}(Y)=\int_{Y^{R}} p_{Y}(x)^{r}\left(x-p_{Y}(x)\right)^{s} d x \in\left(\mathbb{R}^{n}\right)^{\otimes(r+s)} .
$$

Here $p_{Y}(x)$ denotes the point in $Y$ closest to $x$. This is well-defined for almost all $x$.

If $X$ has positive reach and $R<\operatorname{Reach}(X)$, then the Voronoi tensors satisfy the following Steiner formula:

$$
\mathcal{V}_{R}^{r, s}(X)=r ! s ! \sum_{j=0}^{d} \kappa_{j+s} R^{j+s} \Phi_{n-j}^{r, s}(X)
$$

This follows from an application of the generalized Steiner formula in [7].

If the Voronoi tensors are known for $d+1$ distinct values $R_{0}, \ldots, R_{d}$ of $R$, then we get $d+1$ equations:

$$
\left(\begin{array}{c}
\mathcal{V}_{R_{0}}^{r, s}(X) \\
\vdots \\
\mathcal{V}_{R_{d}}^{r, s}(X)
\end{array}\right)=r ! s !\left(\begin{array}{ccc}
\kappa_{s} R_{0}^{s} & \ldots & \kappa_{s+d} R_{0}^{s+d} \\
\vdots & & \vdots \\
\kappa_{s} R_{d}^{s} & \ldots & \kappa_{s+d} R_{d}^{s+d}
\end{array}\right)\left(\begin{array}{c}
\Phi_{d}^{r, s}(X) \\
\vdots \\
\Phi_{0}^{r, s}(X)
\end{array}\right)
$$

The matrix is invertible since it is a product of a diagonal matrix and a Vandermonde matrix, so the system can be solved for the Minkowski tensors. 
The Voronoi tensors of the set $X \cap a \mathbb{L}$ can be computed from the image. If we take this as an estimate for the Voronoi tensors of $X$, we obtain the following estimators for the Minkowski tensors:

$$
\left(\begin{array}{c}
\hat{\Phi}_{d}^{r, s}(X) \\
\vdots \\
\hat{\Phi}_{0}^{r, s}(X)
\end{array}\right)=\frac{1}{r ! s !}\left(\begin{array}{ccc}
\kappa_{s} R_{0}^{s} & \ldots & \kappa_{s+d} R_{0}^{s+d} \\
\vdots & & \vdots \\
\kappa_{s} R_{d}^{s} & \ldots & \kappa_{s+d} R_{d}^{s+d}
\end{array}\right)^{-1}\left(\begin{array}{c}
\mathcal{V}_{R_{0}}^{r, s}(X \cap a \mathbb{L}) \\
\vdots \\
\mathcal{V}_{R_{d}}^{r, s}(X \cap a \mathbb{L})
\end{array}\right)
$$

The following convergence result for these estimators was proved in [6]:

Theorem 10. Suppose $X$ is a topologically regular set of positive reach and $R<$ $\operatorname{Reach}(X)$. Then

$$
\lim _{a \rightarrow 0} \mathcal{V}_{R}^{r, s}(X \cap a \mathbb{L})=\mathcal{V}_{R}^{r, s}(X) .
$$

By linearity in (2.5), we obtain

$$
\lim _{a \rightarrow 0} \hat{\Phi}_{m}^{r, s}(X)=\Phi_{m}^{r, s}(X) .
$$

The Voronoi tensors of $X \cap a \mathbb{L}$ have a simple expression in terms of the Voronoi cells

$$
V_{x}=\left\{y \in \mathbb{R}^{n} \mid \forall z \in(X \cap a \mathbb{L}) \backslash\{x\}:\|x-y\|<\|z-y\|\right\} .
$$

Namely,

$$
\mathcal{V}_{R}^{r, s}(X \cap a \mathbb{L})=\sum_{x \in X \cap a \mathbb{L}} x^{r} \int_{V_{x} \cap B_{x}(R)}(y-x)^{s} d y,
$$

where $B_{x}(R)$ is the ball around $x$ of radius $R$. Thus, in order to compute the estimator, one needs to compute the Voronoi cells of $X \cap a \mathbb{L}$ and do an integral over each of these. This is more computationally involved than the local algorithms, but there exist relatively fast algorithms to compute Voronoi cells.

\section{Grey-scale images}

The black-and-white model for digital images introduced in Section 2.2 is often too idealized for real world images. Due to limitations of the measuring device, the light from a single point will be spread out over the whole space. We are thus unable to measure precisely whether or not a point lies in $X$. Instead, we measure a light intensity. Associating a grey tone to each light intensity, this results in a grey-scale image where we see a blurred zone around the boundary of the object.

Blurring may seem like an obstacle to the estimation of intrinsic volumes. The simplest way to deal with it is to use thresholding, i.e. to choose a threshold value $\beta$ and convert all pixels with grey-value larger than $\beta$ to black and all other pixels to white. The algorithms for black-and-white images may then be applied to the thresholded image. One would expect this to introduce an extra bias. At the same time, a lot of information is thrown away when an image is thresholded. We shall see below that basing algorithms directly on the grey-values can actually be an advantage. 


\subsection{Models for grey-scale images}

Let $\rho: \mathbb{R}^{n} \rightarrow[0, \infty)$ be the point spread function (PSF) that describes how the light originating from a point at the origin is spread out over $\mathbb{R}^{n}$. The intensity $\theta^{X}(x)$ that can be measured at $x \in \mathbb{R}^{n}$ is then an integral of the contributions from all points in $X$ :

$$
\theta^{X}(x)=\int_{X} \rho(x-y) d y .
$$

In other words, $\theta^{X}$ is the convolution of $\mathbf{1}_{X}$ with $\rho$.

We have assumed that the PSF is independent of the position of the point. Moreover, we assume that $\rho$ is bounded, continuous, and that $\int_{\mathbb{R}^{n}} \rho(x) d x=1$. Since the results below only deal with rotation invariant PSF's, we will assume throughout that $\rho(x)=\rho(|x|)$, i.e. the light received from a point depends only on the distance to the point. More general PSF's have been considered in [24].

In applications, the PSF is often modeled by the Gaussian $\rho(x)=(2 \pi)^{-d / 2} e^{-x^{2} / 2}$, which satisfies all the above assumptions.

In a digital grey-scale image, we measure the intensity $\theta^{X}$ at the midpoint of each pixel. That is, the information we have is

$$
\theta_{\mid \mathbb{L}}^{X}: \mathbb{L} \rightarrow[0,1]
$$

We also consider the following transformation of $\rho$ :

$$
\rho_{\varepsilon}(x)=\varepsilon^{-n} \rho\left(\varepsilon^{-1} x\right) .
$$

Small values of $\varepsilon$ correspond to little blurring, meaning that the grey-values are concentrated close to the boundary of $X$. The intensity function corresponding to $\rho_{\varepsilon}$ will be denoted by $\theta_{\varepsilon}^{X}$.

\subsection{Local algorithms for grey-scale images}

Local algorithms for grey-scale images are algorithms based on the local $k \times \cdots \times k$ configurations of grey-values in the image. A $k \times \cdots \times k$ configuration of grey-values is an element of

$$
[0,1]^{C_{0,0}^{k}}=\left\{\left\{\theta_{s}\right\}_{s \in C_{0,0}^{k}} \mid \theta_{s} \in[0,1]\right\} .
$$

We denote the configuration $\left\{\theta_{\varepsilon}^{X}(x)\right\}_{x \in z+a C_{0,0}^{k}}$ of grey-values observed on $z+a C_{0,0}^{k}$ by $\theta_{\varepsilon}^{X}(z, a, k)$. To each configuration we associate a weight. We can think of this as a function $f:[0,1]^{C_{0,0}^{k}} \rightarrow \mathbb{R}$.

Definition 11. A local algorithm for $V_{m}$ is an algorithm of the form

$$
\hat{V}_{m}(X)=a^{n} \varepsilon^{m-n} \sum_{z \in a \mathbb{L}} f\left(\theta_{\varepsilon}^{X}(z, a, k)\right),
$$

where $f:[0,1]^{C_{0,0}^{k}} \rightarrow \mathbb{R}$ is a measurable function.

The factor $a^{n}$ compensates for the growing number of terms in the sum when $a \rightarrow 0$. The factor $\varepsilon^{m-n}$ ensures the right degree of homogeneity. 


\subsubsection{Convergence of grey-scale local algorithms}

We again test the convergence of the algorithms in the design based setting with a stationary lattice. We are interested in convergence when $\varepsilon \rightarrow 0$, i.e. when the blurring becomes small.

We restrict ourselves to estimators with $k=1$. Thus, the weight function is a function $f:[0,1] \rightarrow \mathbb{R}$ and $\hat{V}_{m}$ takes the following simple form

$$
\hat{V}_{m}(X)=a^{n} \varepsilon^{m-n} \sum_{z \in a \mathbb{L}} f\left(\theta_{\varepsilon}^{X}(z)\right) .
$$

The asymptotic behavior of estimators based on larger $k \times \cdots \times k$ configurations is studied in [24], but the results are harder to interpret in this case, so we omit them here.

The mean of an estimator of the form (3.1) is again given by a simple formula:

$$
\begin{aligned}
\mathbf{E} \hat{V}_{m}(X) & =a^{n} \varepsilon^{m-n} \mathbf{E}\left(\sum_{z \in a \mathbb{L}} f\left(\theta_{\varepsilon}^{X}(z)\right)\right) \\
& =a^{n} \varepsilon^{m-n} \int_{C_{0}^{1}}\left(\sum_{z \in a \mathbb{L}_{0}} f\left(\theta_{\varepsilon}^{X}(z+a c)\right)\right) d c \\
& =\varepsilon^{m-n} c_{\mathbb{L}}^{-1} \int_{\mathbb{R}^{n}} f \circ \theta_{\varepsilon}^{X}(z) d c .
\end{aligned}
$$

We must study the asymptotic behavior of this integral. To do so, we introduce a function $\theta: \mathbb{R} \rightarrow[0,1]$ that will appear in the results below. This is given by

$$
\theta(t)=\int_{\mathbb{R}^{n}} \mathbf{1}_{\{\langle x, u\rangle \leq 0\}} \rho(t u-x) d x
$$

where $u \in S^{n-1}$ is a unit vector. By rotation invariance of $\rho, \theta$ is independent of $u$.

The map $t \mapsto \theta(t)$ is the intensity function of a halfspace perpendicular to $u$ measured at a point of signed distance $t$ from the boundary of the halfspace. If we zoom in on the boundary of a sufficiently smooth set, it will look almost like a halfspace. Therefore, the blurred image will locally look almost like a blurred halfspace when $\varepsilon$ is small. This is the intuitive reason why $\theta$ shows up in the limit $\varepsilon \rightarrow 0$.

The theorem is stated under the assumption that $X$ is a gentle set. This is a mild smoothness condition ensuring that almost every boundary point has a well-defined tangent space. It is satisfied by all finite unions elements from $\mathcal{K}_{0}^{n}$ and all sets of positive reach. See [10] for the precise definition.

We can now state the following convergence result for surface area estimators:

Theorem 12. Let $X$ be a gentle set. Suppose $f:[0,1] \rightarrow \mathbb{R}$ is continuously differentiable on the interval $[\beta, \omega] \subseteq(0,1)$ and that $f$ is zero outside $[\beta, \omega]$. Then

$$
\lim _{\varepsilon \rightarrow 0} \mathbf{E} \hat{V}_{n-1}(X)=c_{1}(f, \rho) V_{n-1}(X),
$$

where

$$
c_{1}(f, \rho)=2 c_{\mathbb{L}}^{-1} \int_{\mathbb{R}} f \circ \theta(t) d t .
$$


If $f>0$ on $(\beta, \omega)$, then $c_{1}(f, \rho) \neq 0$. In this case,

$$
c_{1}(f, \rho)^{-1} \hat{V}_{n-1}(X)
$$

is an asymptotically unbiased estimator for $V_{n-1}(X)$.

Similarly, there is a result for estimation of integrated mean curvature:

Theorem 13. Let $X$ be an $r$-regular set and assume that $\rho$ has compact support. Suppose $f:[0,1] \rightarrow \mathbb{R}$ is continuously differentiable on $[\beta, 1-\beta] \subset(0,1)$ and zero outside $[\beta, 1-\beta]$. If $f(t)=-f(1-t)$, then

$$
\lim _{\varepsilon \rightarrow 0} \hat{V}_{n-2}(X)=c_{2}(f, \rho) V_{n-2}(X) .
$$

\subsubsection{Some examples}

To illustrate the results, we can look at some simple examples of local algorithms for grey-scale images. The simplest algorithm for surface area is of the form (3.1) with $f=\mathbf{1}_{[\beta, 1-\beta]}$, i.e.

$$
\hat{V}_{n-1}(X)=a^{n} \varepsilon^{-1} \sum_{z \in a \mathbb{L}} \mathbf{1}_{[\beta, 1-\beta]}\left(\theta_{\varepsilon}^{X}(z)\right)
$$

Up to a factor $a^{n} \varepsilon^{-1}$, this is the number of pixels with grey-value in the interval $[\beta, 1-\beta]$. According to Theorem 2, the mean of this estimator is $\varepsilon^{-1}$ times the volume of the band around bd $X$ with grey-values in $[\beta, 1-\beta]$. Intuitively, the volume of this a band should be proportional to $\varepsilon V_{n-1}(X)$. Indeed, Theorem 12 shows that

$$
\lim _{a \rightarrow 0} \mathbf{E} \hat{V}_{n-1}(X)=c_{1}(\beta, \rho) V_{n-1}(X),
$$

where

$$
c_{1}(\beta, \rho)=4 c_{\mathbb{L}}^{-1} \theta^{-1}(\beta) .
$$

When $\rho$ is the standard Gaussian PSF, $\theta$ is the distribution function of a standard normal distribution on $\mathbb{R}$, so $c_{1}(\beta, \rho)$ can be computed directly. If the PSF is unknown, it may be necessary to determine $c_{1}(\beta, \rho)$ experimentally.

This algorithm is extremely simple, since it only requires thresholding at two different levels and computing the difference in the number of black lattice points. However, other algorithms for surface area could also be worth considering. For instance, it might be relevant to choose a function that puts more emphasis on grey-values close to $1 / 2$ since these are expected to lie close to bd $X$.

For estimation of $V_{n-2}$, one can consider the weight function $f=\mathbf{1}_{[\beta, 1 / 2]}-\mathbf{1}_{[1 / 2,1-\beta]}$. The resulting algorithm is given by counting the number of grey-values between $\beta$ and $1 / 2$ and subtracting the number of grey-values between $1 / 2$ and $1-\beta$. For suitable $\beta$, the constant $c_{2}(f, \rho)$ in Theorem 13 will be non-zero and hence we can divide by it to get an estimator for $V_{n-2}$. 


\subsubsection{Variance of local algorithms for grey-scale images}

The above convergence results hold in any resolution $a$. This may seem a bit counterintuitive. If $\varepsilon$ is small, which is necessary to obtain good precision, the grey-values in the interval $[\beta, \omega]$ are concentrated in a very narrow band around $b d X$. If, at the same time, the resolution is low, then it is likely that the lattice does not intersect this band. Thus we expect to see large deviations from the mean. This is captured by the variance, as shown in [25]:

Theorem 14. Suppose that $X$ is a smooth $n$-dimensional manifold with boundary in dimension $n>1$ and that $\rho$ and $f$ are smooth functions. Let $\mathbb{L}$ be a stationary isotropic lattice. Then there is a constant $C(X, \rho, f)>0$ such that for all a and $\varepsilon$ sufficiently small,

$$
\operatorname{var}\left(\hat{V}_{n-1}(X)\right) \leq C(X, \rho, f) a^{n} \varepsilon^{-1} .
$$

The interesting thing here is that the effect of the resolution on the variance is much larger than the effect of $\varepsilon$. In particular, if $a$ and $\varepsilon$ tend to 0 at the same rate, the variance will also go to zero. So to obtain small variance, it is more important to have high resolution than little blurring.

A computation similar to the one in Section 2.2.5 with $\varepsilon^{-1} f \circ \theta_{\varepsilon}^{X}$ replacing $c_{\mathbb{L}} \mathbf{1}_{X}$ shows that

$$
\operatorname{var}\left(\hat{V}_{n-1}(X)\right)=\varepsilon^{-2} \omega_{n}^{-1} c_{\mathbb{L}}^{-2} \sum_{z \in \mathbb{L}_{0}^{*} \backslash\{0\}} \int_{S^{n-1}}\left|\mathcal{F}\left(f \circ \theta_{\varepsilon}^{X}\right)\left(a^{-1}|z| u\right)\right|^{2} d u
$$

As in the case of volume estimators, the variance can be studied by considering the Fourier coefficients in this sum. This is done in [25].

\subsection{Minkowski tensors from grey-scale images}

As in the black-and-white case, local estimators for Minkowski tensors would have to take the position of each configuration into account. That is, we must consider estimators of the form

$$
a^{n} \varepsilon^{-m} \sum_{z \in a \mathbb{L}} f\left(z, \theta_{\varepsilon}^{X}(z, a, k)\right),
$$

where $f: \mathbb{R}^{n} \times[0,1]^{C_{0,0}^{k}} \rightarrow\left(\mathbb{R}^{n}\right)^{\otimes(r+s)}$. Such estimators were studied in [26] with the purpose of defining estimators for Minkowski tensors. Under the assumption that $a=\varepsilon$ and $X$ is $r$-regular, it was shown that all Minkowski tensors of the form $\Phi_{n-1}^{r, s}(X)$ and $\Phi_{n-2}^{r, 0}(X)$ can be estimated by an asymptotically unbiased algorithm of this form. This requires that the point spread function is known.

We will not show this in general here but just outline the idea for surface tensors in $2 \mathrm{D}$. We assume that $\mathbb{L}$ is the standard lattice $\mathbb{Z}^{2}$ spanned by the basis vectors $e_{1}$ and $e_{2}$. Consider the estimator

$$
\hat{\Phi}_{n-1}^{r, s}(X)=a^{n-1} \sum_{z \in a \mathbb{Z}^{2}} z^{r} f\left(\theta_{a}^{X}(z, a, 2)\right) .
$$

Comparing with the definition (1.1), we expect that $f$ should be an estimate for $u^{s}$. 
a.

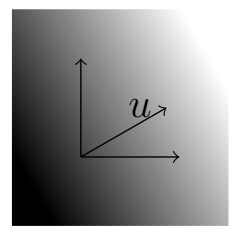

b.

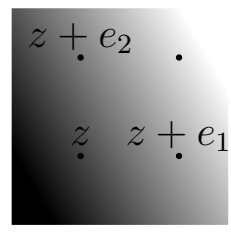

Figure 3: a. A blurred halfspace with normal $u$. The normal vector is determined by how fast the grey-values change in the horizontal and vertical direction. b. A configuration of grey-values. To determine $u$ one can look at how the grey-vales change from $z$ to $z+e_{1}$ and from $z$ to $z+e_{2}$.

The idea is to estimate the normal direction $u$ by the direction in which the grey-values decrease fastest. Given a $2 \times 2$ configuration, we can look at how fast the grey-values change in the vertical and horizontal direction to get an idea about the normal direction, see Figure 3.

More precisely, consider a boundary point $x$ with normal vector $u$ and suppose that $a$ is small. Then in a neighborhood around $x$, the image will look almost like a blurred halfspace with normal vector $u$. In particular, if $y$ lies in this neighborhood, then the grey-value at $y$ will be approximately $\theta(\langle y-x, u\rangle)$ since $\theta(t)$ is the grey-value of a point at signed distance $t$ to the boundary of the halfspace.

If the whole $2 \times 2$ lattice block $\left.z+a C_{0,0}^{2}=\left\{z, z+a e_{1}, z+a e_{2}\right), z+a e_{1}+a e_{2}\right\}$ lies in this neighborhood of $x$, then

$$
\begin{aligned}
\theta_{a}^{X}(z) & \approx \theta(\langle z-x, u\rangle), \\
\theta_{a}^{X}\left(z+a e_{1}\right) & \approx \theta\left(\left\langle z+a e_{1}-x, u\right\rangle\right), \\
\theta_{a}^{X}\left(z+a e_{2}\right) & \approx \theta\left(\left\langle z+a e_{2}-x, u\right\rangle\right) .
\end{aligned}
$$

It follows that

$$
\begin{aligned}
& \theta^{-1}\left(\theta_{a}^{X}\left(z+a e_{1}\right)\right)-\theta^{-1}\left(\theta_{a}^{X}(z)\right) \approx\left\langle a e_{1}, u\right\rangle=a u_{1}, \\
& \theta^{-1}\left(\theta_{a}^{X}\left(z+a e_{2}\right)\right)-\theta^{-1}\left(\theta_{a}^{X}(z)\right) \approx\left\langle a e_{2}, u\right\rangle=a u_{2},
\end{aligned}
$$

where $u=\left(u_{1}, u_{2}\right)$. We may use the left hand side to estimate $u$ and estimate $u^{s}$ from this. Note that this requires that $\theta$, which is determined by the point spread function, is known. The convergence of this algorithm is shown in [26].

\section{Acknowledgements}

This research was supported by Centre for Stochastic Geometry and Advanced Bioimaging, funded by a grant from the Villum Foundation.

\section{References}

[1] Brandolini, L., Hofmann, S., Iosevich, A.: Sharp rate of average decay of the Fourier transform of a bounded set. Geom. Funct. Anal. 13, 671-680 (2003) 
[2] Chazelle, B.: An optimal convex hull algorithm in any fixed dimension. Discrete Comput. Geom. 10, no. 4, 377-409 (1993)

[3] Christensen, S.T., du Plessis, A.: Reconstruction of $r$-regular surfaces. (In preparation)

[4] Herz, C.S.: Fourier transforms related to convex sets. Ann. of Math. 75, 81-92 (1962)

[5] Hörrmann, J., Svane, A.M.: Local digital algorithms applied to Boolean models. (In preparation)

[6] Hug, D., Kiderlen, M., Svane, A.M.: Voronoi-based estimation of Minkowski tensors. (In preparation)

[7] Hug, D., Last, G., Weil, W.: A local Steiner-type formula for general closed sets and applications. Math. Z. 246, no. 1-2, 237-272 (2004)

[8] Kampf, J.: A limitation of the estimation of intrinsic volumes via pixel configuration counts. Mathematika 60, no. 2, 485-511 (2014)

[9] Kiderlen, M.: Estimating the Euler characteristic of a planar set from a digital image. Journal of Visual Communication and Image Representation 17, 1237-1255 (2006)

[10] Kiderlen, M., Rataj, J.: On infinitesimal increase of volumes of morphological transforms. Mathematika 53, no. 1, 103-127 (2006)

[11] Kiêu, K., Mora, M.: Precision of stereological planar area predictors, J. Microsc. 222, 201-211 (2006)

[12] Klenk, S., Schmidt, V., Spodarev, E.: A new algorithmic approach to the computation of Minkowski functionals of polyconvex sets. Comput. Geom. 34, no. 3, 127-148 (2006)

[13] Klette, R., Rosenfeld, A.: Digital Geometry. Elsevier, San Francisco (2004)

[14] Ohser, J., Mücklich, F.: Statistical Analysis of Microstructures. John Wiley \& Sons, Chichester (2000)

[15] Ohser, J., Nagel, W., Schladitz, K.: Miles formulae for Boolean models observed on lattices. Image Anal. Stereol. 28, no. 2, 77-92 (2009)

[16] Pavlidis, T.: Algorithms for Graphics and Image Processing. Computer Science Press (1982)

[17] Schneider, R.: Convex bodies: The Brunn-Minkowski Theory. Cambridge University Press, Cambridge (1993)

[18] Schröder-Turk, G.E., Kapfer, S.C., Breidenbach, B., Beisbart, C., Mecke, K.: Tensorial Minkowski functionals and anisotropy measures for planar patterns. J. Microsc. 238, $57-74(2008)$

[19] Schröder-Turk, G.E., Mickel, W., Kapfer, S.C., Schaller, F.M., Breidenbach, B., Hug, D., Mecke, K.: Minkowski tensors of anisotropic spatial structure. New Journal of Physics 15, (8), 083028 (2013)

[20] Stein, E.M., Weiss, G.: Introduction to Fourier Analysis. Princeton University Press, Princeton (1971) 
[21] Svane, A.M.: Local digital estimators of intrinsic volumes for Boolean models and in the design based setting. Adv. Appl. Prob. 46, 35-58 (2014)

[22] Svane, A.M.: Local digital algorithms for estimating the integrated mean curvature of $r$-regular sets. To appear in: Discrete Comput. Geom.

[23] Svane, A.M.: On multigrid convergence of local algorithms for intrinsic volumes. J. Math. Imaging Vis. 49, no. 1, 352-376 (2014)

[24] Svane, A.M.: Estimation of intrinsic volumes from digital grey-scale images. J. Math. Imaging Vis. 49, no. 2, 148-72 (2014)

[25] Svane, A.M.: Asymptotic variance of grey-scale surface area estimators. Adv. in Appl. Math. 62, 41âĂŞ73 (2015)

[26] Svane, A.M.: Estimation of Minkowski tensors from digital grey-scale images. Image Anal. Stereol. 34, no. 1, 51-61 (2015)

[27] Zähle, M.: Integral and current representation of Federer's curvature measures. Arch. Math. (Basel) 46, 557-567 (1986)

[28] Ziegel, J., Kiderlen, M.: Estimation of surface area and surface area measure of threedimensional sets from digitizations. Image Vis. Comput. 28, 64-77 (2010) 